\title{
Astrochemical relevance of VUV ionization of large PAH cations ${ }^{\star}$
}

\author{
G. Wenzel ${ }^{1}$, C. Joblin ${ }^{1}$, A. Giuliani ${ }^{2,3}$, S. Rodriguez Castillo ${ }^{1,4}$, G. Mulas ${ }^{1,5}$, M. Ji $^{1}$, H. Sabbah $^{1,6}$, S. Quiroga ${ }^{7}$, \\ D. Peña ${ }^{7}$, and L. Nahon ${ }^{2}$
${ }^{1}$ Institut de Recherche en Astrophysique et Planétologie (IRAP), Université de Toulouse (UPS), CNRS, CNES, 9 Avenue du Colonel Roche, 31028 Toulouse, France e-mail: christine.joblin@irap.omp.eu
2 Synchrotron SOLEIL, L'Orme des Merisiers, 91192 Saint Aubin, Gif-sur-Yvette, France
3 INRAE, UAR1008, Transform Department, Rue de la Géraudière, BP 71627, 44316 Nantes, France
${ }^{4}$ Laboratoire de Chimie et Physique Quantiques (LCPQ/IRSAMC), Université de Toulouse (UPS), CNRS, 118 Route de Narbonne, 31062 Toulouse, France
${ }^{5}$ Istituto Nazionale di Astrofisica - Osservatorio Astronomico di Cagliari, Via della Scienza 5, 09047 Selargius (CA), Italy
${ }^{6}$ Laboratoire Collisions Agrégats Réactivité (LCAR/IRSAMC), Université de Toulouse (UPS), CNRS, 118 Route de Narbonne, 31062 Toulouse, France
${ }^{7}$ Centro de Investigación en Química Biolóxica e Materiais Moleculares (CiQUS) and Departamento de Química Orgánica, Universidade de Santiago de Compostela, 15782 Santiago de Compostela, Spain

Received 9 April 2020 / Accepted 1 July 2020

\begin{abstract}
Context. As part of interstellar dust, polycyclic aromatic hydrocarbons (PAHs) are processed by an interaction with vacuum ultraviolet (VUV) photons emitted by hot stars. This interaction leads to the emission of not only the well-known aromatic infrared bands, but also electrons, which can significantly contribute to the heating of the interstellar gas.

Aims. Our aim is to investigate the impact of molecular size on the photoionization properties of cationic PAHs.

Methods. Trapped PAH cations of sizes between 30 and 48 carbon atoms were submitted to VUV photons in the range of 9-20 eV from the DESIRS beamline at the synchrotron SOLEIL. All resulting photoproducts including dications and fragment cations were mass-analyzed and recorded as a function of photon energy.

Results. Photoionization is found to be predominant over dissociation at all energies, which differs from the conclusions of an earlier study on smaller PAHs. The photoionization branching ratio reaches 0.98 at $20 \mathrm{eV}$ for the largest studied PAH. The photoionization threshold is observed to be between 9.1 and $10.2 \mathrm{eV}$, in agreement with the evolution of the ionization potential with size. Ionization cross sections were indirectly obtained and photoionization yields extracted from their ratio with theoretical photoabsorption cross sections, which were calculated using time-dependent density functional theory. An analytical function was derived to calculate this yield for a given molecular size.

Conclusions. Large PAH cations could be efficiently ionized in $\mathrm{H}$ I regions and contribute to the heating of the gas by the photoelectric effect. Also, at the border of or in $\mathrm{H}$ II regions, PAHs could be exposed to photons of energy higher than $13.6 \mathrm{eV}$. Our work provides recipes to be used in astronomical models to quantify these points.
\end{abstract}

Key words. astrochemistry - methods: laboratory: molecular - molecular processes - ISM: molecules - dust, extinction ultraviolet: ISM

\section{Introduction}

Polycyclic aromatic hydrocarbons (PAHs) play a major role in the physics and chemistry of photodissociation regions (PDRs). They strongly absorb the vacuum ultraviolet (VUV) photons emitted by hot stars and relax by emission in the aromatic infrared bands (AIBs). The interaction with VUV photons can lead to other relaxation processes including ionization and dissociation. All these processes together with reactive processes involving in particular electrons and hydrogen $\left(\mathrm{H}, \mathrm{H}_{2}\right)$ govern the evolution of the PAH population in the diffuse interstellar medium (Le Page et al. 2003), in circumstellar disks (Visser et al. 2007), and in reflection nebulae (Montillaud et al. 2013). The results of these chemical models suggest that large PAHs with a typical carbon number, $N_{\mathrm{C}}$, of 50 or more dominate the AIB emission, which led to the grandPAH hypothesis that large and

\footnotetext{
* The dataset associated with this work can be found under https://doi.org/10.5281/zenodo. 3899775
}

possibly compact PAHs dominate the emission in bright PDRs (Andrews et al. 2015). In some regions associated with these PDRs, large PAHs are expected to be ionized, even reaching the dicationic stage (Tielens 2005; Andrews et al. 2016).

In a previous study we investigated the branching ratio between ionization and fragmentation upon VUV irradiation for medium-sized PAH cations (Zhen et al. 2016) with an $N_{\mathrm{C}}$ of between 16 and 24 . For all of these cations, fragmentation was observed to be the dominant channel at least up to a photon energy of $13.6 \mathrm{eV}$ which is relevant for $\mathrm{H}$ I regions. In the case of larger PAH cations, ionization is expected to be by far the dominant channel as suggested by the study of the hexa-perihexabenzocoronene (HBC) cation, $\mathrm{C}_{42} \mathrm{H}_{18}^{+}$, by Zhen et al. (2015). Here, our objective is to quantify the growing importance of ionization as the molecular size increases. Following Zhen et al. (2016), we studied the photoprocessing of PAH cations with an $N_{\mathrm{C}}$ between 30 and 48 atoms over the 9.5-20.0 eV VUV range. Photon energies above the Lyman limit are relevant to PAHs 


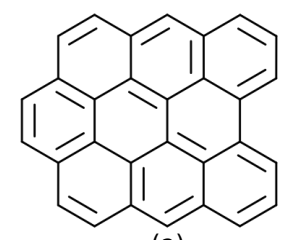

(a)
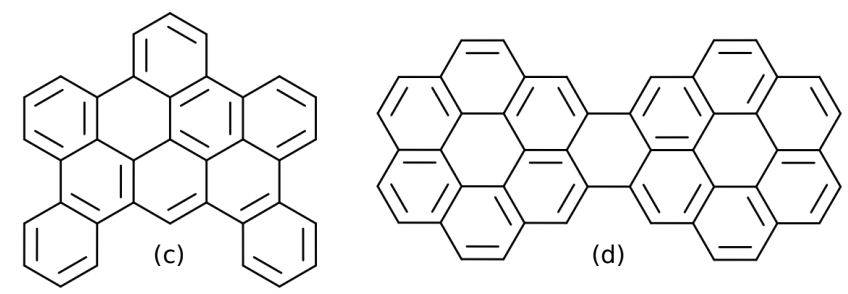

Fig. 1. Molecular structures of the studied PAHs, namely $(a)$ benzobisanthene, $\mathrm{C}_{30} \mathrm{H}_{14}^{+}$, (b) ovalene, $\mathrm{C}_{32} \mathrm{H}_{14}^{+}$, (c) DBPP, $\mathrm{C}_{36} \mathrm{H}_{18}^{+}$, and $(d)$ dicoronylene, $\mathrm{C}_{48} \mathrm{H}_{20}^{+}$.

observed at the border of ionization fronts in PDRs (Vicente et al. 2013), as well as in H II regions (Compiègne et al. 2007).

Although we also report the branching ratio between ionization and dissociation here, our analysis is focused on ionization. More specifically, we derive the photoionization yield, which is important to model the charge balance of PAHs and its impact on the AIB spectrum (Bakes et al. 2001), but also to evaluate the contribution of these species to the photoelectric heating rate (Bakes \& Tielens 1994a; Weingartner \& Draine 2001a). The experimental method is described in Sect. 2 and the results are presented in Sect. 3. In Sect. 4, we discuss the astrophysical implications and propose recipes to be used in astrophysical models. We conclude in Sect. 5.

\section{Experimental method and data analysis}

We have used the Thermo Scientific ${ }^{\mathrm{TM}}$ LTQ XL ${ }^{\mathrm{TM}}$ linear ion trap (LTQ ion trap) as described in Milosavljević et al. (2012), which is available at the VUV beamline DESIRS at the synchrotron SOLEIL (Nahon et al. 2012).

The production of PAH cations in the LTQ ion trap was performed using an atmospheric pressure photoionization (APPI) source which required the species of interest to be in solution before their injection with a syringe. This part was a major limitation on the size range of PAHs that we were able to study due to the insolubility of large PAHs. In the present study, we were able to investigate four large $\mathrm{PAH}$ cations with $N_{\mathrm{C}}$ ranging from 30 to 48 , namely (a) benzobisanthene, $\mathrm{C}_{30} \mathrm{H}_{14}^{+}$, (b) ovalene, $\mathrm{C}_{32} \mathrm{H}_{14}^{+}$, (c) dibenzophenanthropentaphene (DBPP), $\mathrm{C}_{36} \mathrm{H}_{18}^{+}$, and (d) dicoronylene, $\mathrm{C}_{48} \mathrm{H}_{20}^{+}$. Sample (b) originated from Janssen Chimica (Belgium), and samples (a) and (c) from the PAH Research Institute in Greifenberg (Dr. Werner Schmidt). The synthesis of compound (d) is briefly reported in Appendix A. The molecular structures of the studied species are depicted in Fig. 1. Emptying the syringe was performed at a constant flow rate for each experiment: $4 \mu \mathrm{lmin}^{-1}$ for compounds (a) and (b), $6 \mu \mathrm{lmin}^{-1}$ for compound (c), and $10 \mu \mathrm{min}^{-1}$ for compound (d). The presence of UV irradiation from a $\mathrm{Kr}$ discharge lamp ensured a soft creation of PAH cations without fragmentation (Giuliani et al. 2012). The formed cations were then guided through ion optics into the LTQ ion trap in which a constant He pressure of $p \approx 10^{-3}$ mbar was held. The ions were cooled by the collisions with $\mathrm{He}$ atoms, and the PAH cations of interest, the so-called parent ions, were isolated through specific mass selection and ejection of other species from the ion trap including the ${ }^{13} \mathrm{C}$ isotopomers.

The parent ions were then submitted to the VUV synchrotron radiation which was tuned from 9.5 to $20.0 \mathrm{eV}$ in steps of $0.1,0.2,0.3$, or $0.5 \mathrm{eV}$ depending on the photon energy range, with the exception of $\mathrm{C}_{30} \mathrm{H}_{14}^{+}$, for which we were able to scan only at low energies up to $15.5 \mathrm{eV}$. Higher harmonics of the VUV undulator synchrotron radiation with photon energies lower than $16.0 \mathrm{eV}$ were filtered out by a gas filter filled with Ar gas to a pressure of 0.23 mbar. Above $16.0 \mathrm{eV}$, no such gas filtering is necessary. The photon flux was measured with an IRD AXUV100 calibrated Si photodiode for a monochromator exit slit width of $200 \mu \mathrm{m}$ and was between 0.8 and $2.8 \times 10^{12}$ photons $\mathrm{s}^{-1}$ over the studied photon energy range. A typical photon flux can be derived using a previous calibration of the beam size as a function of photon energy (Douix et al. 2017), yielding values of $1.5-5.210^{14}$ photons $\mathrm{cm}^{-2} \mathrm{~s}^{-1}$. In order to limit possible two-photon consecutive absorption processes, we tuned the photon flux by changing (i) the irradiation time from 0.8 to $0.2 \mathrm{~s}$ for the lower and higher photon energy ranges, respectively, and (ii) the monochromator exit slit width from $200 \mu \mathrm{m}$ at low energies to $70 \mu \mathrm{m}$ at high energies, except for dicoronylene for which values of 400 and $100 \mu \mathrm{m}$ at low and high energies were used, respectively, to improve the signal-to-noise ratio $(\mathrm{S} / \mathrm{N})$. The photon dose was assumed to be linearly proportional to both the irradiation time and the monochromator exit slit width. The probability of two-photon absorption processes could be estimated on the formation of triply charged parent ions, yielding only very small relative intensities below $2 \%$ of the total number of photoproducts.

Depending on the acquisition time, a few hundred mass spectra were recorded at each photon energy and averaged to yield one mass spectrum per photon energy step. Following the same procedure, we also recorded blank mass spectra at each photon energy by selecting a mass close to but sufficiently different from each parent ion. This allowed us to perform background subtraction which eliminates contamination peaks from the mass spectra. The averaging procedure provides us with a statistical standard error (see Appendix D). As an example, the background-subtracted mass spectra for ovalene, which has a mass-to-charge ratio of $m / z=398$, are depicted in Fig. 2 at two different photon energies of $9.5 \mathrm{eV}$ and $15.5 \mathrm{eV}$. The parent ion, $\mathrm{C}_{32} \mathrm{H}_{14}^{+}$, is well isolated; the ${ }^{13} \mathrm{C}$ isotopic parent ion has a residual contribution of less than $1 \%$ remaining in the ion trap. By increasing the photon energy, three different secondary ions can be observed and unambiguously separated, namely the $\mathrm{H}$ and $2 \mathrm{H} / \mathrm{H}_{2}$ loss, and the main doubly ionized parent ion channels. For the presented example of the ovalene cation, $\mathrm{C}_{32} \mathrm{H}_{14}^{+}$, these species are $\mathrm{C}_{32} \mathrm{H}_{13}^{+}, \mathrm{C}_{32} \mathrm{H}_{12}^{+}$, and $\mathrm{C}_{32} \mathrm{H}_{14}^{2+}$, respectively (see Fig. 2). When extracting the peak intensities as in the following, one has to consider the detector gain efficiency that varies with the charge and the mass of the ions of interest. Recommended scaling factors were therefore applied (see Appendix B).

\section{Results and discussion}

\subsection{Action spectra and branching ratio}

The action spectra are determined following the procedure described in Appendix B yielding relative intensities of the photoproducts as used in a previous study (Zhen et al. 2016). The resulting spectra for the photoionization (dication, denoted I) and photodissociation (fragments, denoted F) channels of the four studied PAH cations are shown in Fig. 3 as a function of the 

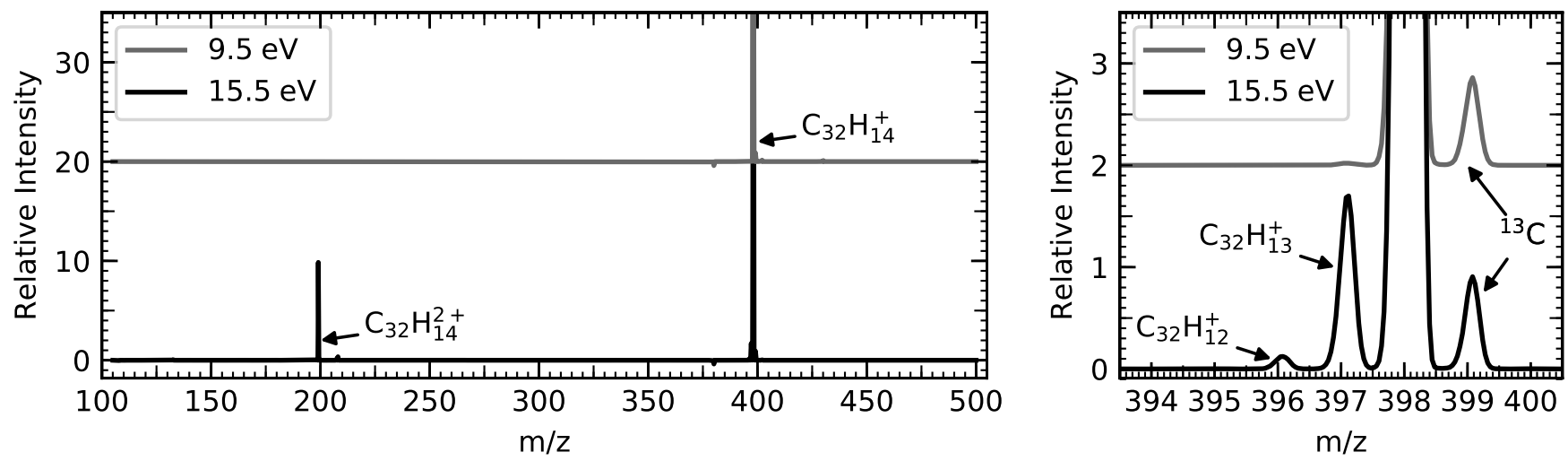

Fig. 2. Mass spectra of the ovalene parent cation, $\mathrm{C}_{32} \mathrm{H}_{14}^{+}, m / z=398$, at two different photon energies. At $9.5 \mathrm{eV}$, none of the photoionization or photodissociation channels are opened whereas at $15.5 \mathrm{eV}$, the doubly ionized parent ion, $\mathrm{C}_{32} \mathrm{H}_{14}^{2+}$, at $m / z=199$ is observed as well as the photofragments due to the loss of one and two hydrogen atoms, $\mathrm{C}_{32} \mathrm{H}_{13}^{+}$and $\mathrm{C}_{32} \mathrm{H}_{12}^{+}$at $m / z=397$ and 396 , respectively.

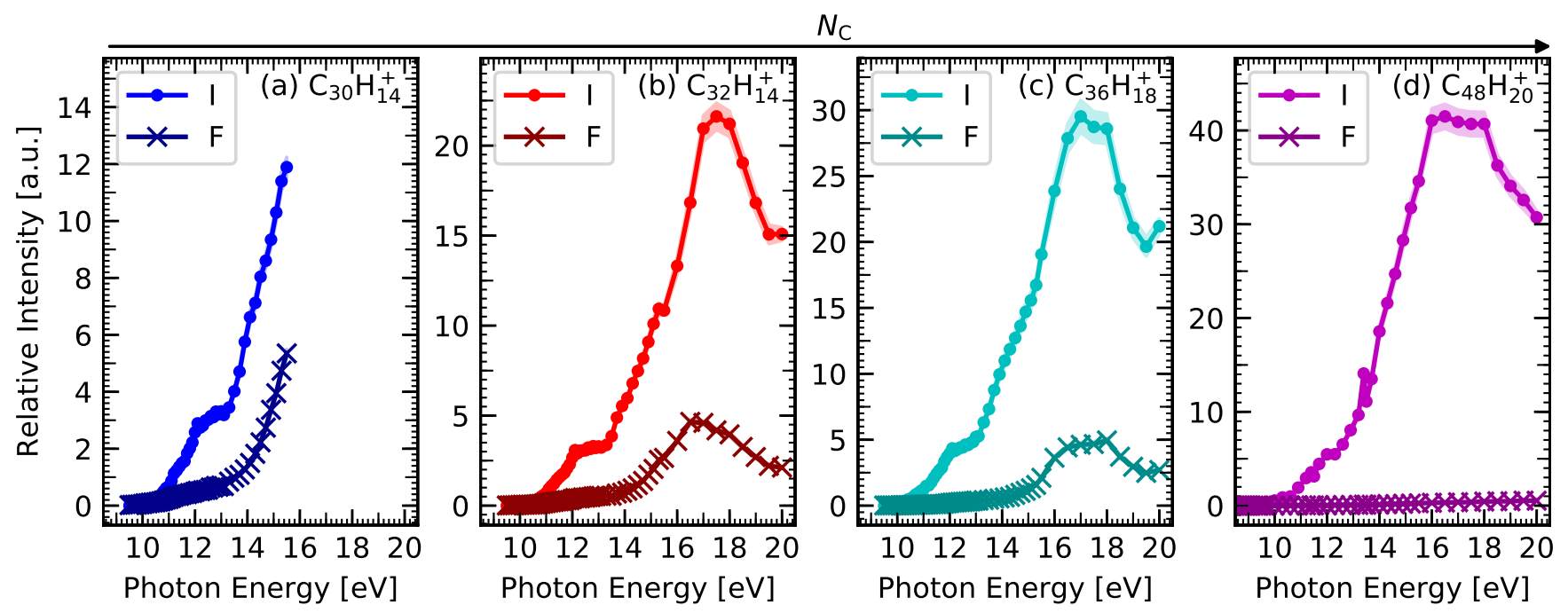

Fig. 3. Action spectra of the photoproducts, dications (I) and fragments $(\mathrm{F})$, as a function of photon energy for all studied PAH cations, $(a)$ benzobisanthene, $(b)$ ovalene, $(c)$ DBPP, and $(d)$ dicoronylene after absorption of a VUV photon. Relative intensities as explained in Appendix B.

photon energy. The $\mathrm{F}$ channel remains small for all investigated $\mathrm{PAH}$ cations at all photon energies and is barely notable for the dicoronylene cation in Fig. 3. More specifically, Fig. 4 shows that the branching ratio (BR) for photoionization relative to photodissociation increases significantly with increasing $N_{\mathrm{C}}$ with a minimal value of 0.98 in the case of the largest studied cation. This trend of large PAHs differs from what was observed in our earlier study of medium-sized PAH cations for which a larger fraction of fragments was observed (Zhen et al. 2016), but is in line with the ionization $\mathrm{BR}$ of about 0.97 at $20 \mathrm{eV}$ derived by Zhen et al. (2015) for the $\mathrm{HBC}$ cation, $\mathrm{C}_{42} \mathrm{H}_{18}^{+}$, by operating their home-made ion trap setup at the DESIRS beamline. These latter authors also reported a value of $0.70 \pm 0.10$ for the ionization $\mathrm{BR}$ of the ovalene cation at $20 \mathrm{eV}$, which can be compared to a value of $0.87 \pm 0.02$ in our experiment. This difference can be interpreted by the low mass resolution achieved in the former experiments which impacted both the isolation of the ${ }^{12} \mathrm{C}$ parent isotopomer before irradiation and the quantification of the abundance of $-\mathrm{H}$ fragments in the photoproducts.

From Fig. 3, we derived appearance energies for the formation of $\mathrm{PAH}^{2+}$ from $\mathrm{PAH}^{+}, \mathrm{AE}^{2+}$. The values are listed in Table 1 and compared to the corresponding computed values for the adiabatic ionization potentials, $\mathrm{IP}^{2+}$, which are extracted from the

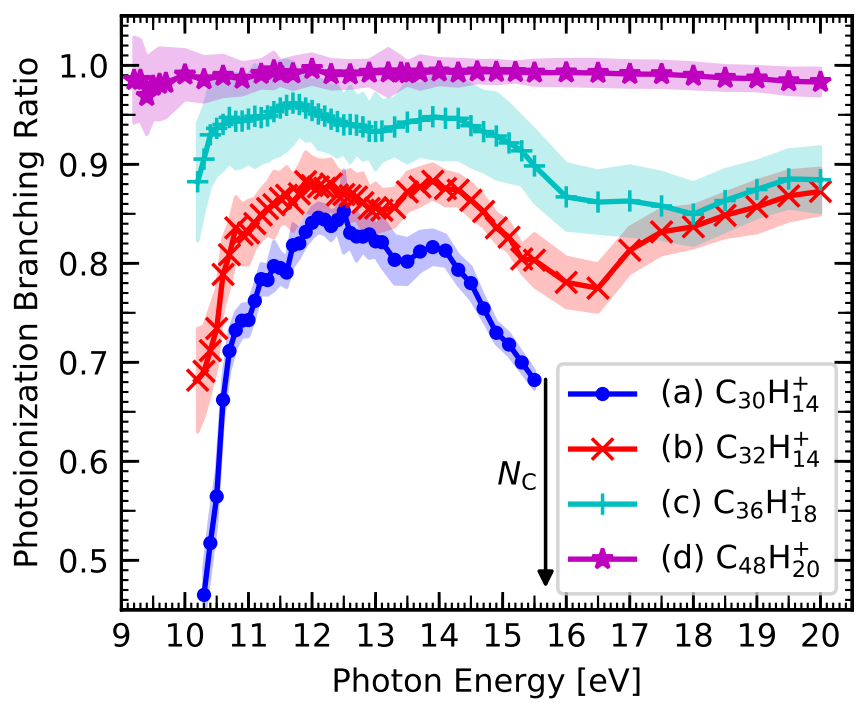

Fig. 4. Photoionization branching ratio relative to photodissociation as a function of photon energy, for (a) benzobisanthene, $\mathrm{C}_{30} \mathrm{H}_{14}^{+}$, (b) ovalene, $\mathrm{C}_{32} \mathrm{H}_{14}^{+}$, (c) DBPP, $\mathrm{C}_{36} \mathrm{H}_{18}^{+}$, and (d) dicoronylene, $\mathrm{C}_{48} \mathrm{H}_{20}^{+}$, after absorption of a single VUV photon. 
Table 1. For doubly ionized $\mathrm{PAH}$ cations, $\mathrm{PAH}^{+} \rightarrow \mathrm{PAH}^{2+}$, values of the theoretical adiabatic ionization potentials, $\mathrm{IP}^{2+}$, and measured appearance energies, $\mathrm{AE}^{2+}$.

\begin{tabular}{lccc}
\hline \hline Formula & $\mathrm{IP}^{2+}[\mathrm{eV}]$ & $\mathrm{AE}^{2+}[\mathrm{eV}]$ & $\mathrm{AE}^{3+}[\mathrm{eV}]$ \\
\hline $\mathrm{C}_{30} \mathrm{H}_{14}^{+}$ & $9.66^{(a)}$ & $10.2 \pm 0.1$ & $14.1 \pm 0.2$ \\
$\mathrm{C}_{32} \mathrm{H}_{14}^{+}$ & $9.82^{(a)}$ & $10.0 \pm 0.1$ & $13.9 \pm 0.2$ \\
$\mathrm{C}_{36} \mathrm{H}_{18}^{+}$ & $9.94^{(b)}$ & $10.0 \pm 0.1$ & $13.9 \pm 0.2$ \\
$\mathrm{C}_{48} \mathrm{H}_{20}^{+}$ & $8.84^{(a)}$ & $9.1 \pm 0.2$ & $-{ }^{(c)}$ \\
\hline
\end{tabular}

Notes. We also list here our recorded $\mathrm{AE}^{3+}$ as obtained from the ionization of $\mathrm{PAH}^{2+} \rightarrow \mathrm{PAH}^{3+}$. ${ }^{(a)}$ Taken from Malloci et al. (2007a). ${ }^{(b)}$ Calculated for this work according to Malloci et al. (2007b). ${ }^{(c)}$ Trication peak out of mass range.

Theoretical Spectral Database of PAHs ${ }^{1}$ (Malloci et al. 2007a) or calculated at the same level of theory for the missing $\mathrm{IP}^{2+}$ of the DBPP cation according to Malloci et al. (2007b). Experimental and theoretical values are found to be in good agreement considering the accuracy of $\sim 0.3 \mathrm{eV}$ for the calculated values. The trend of a slow decrease of $\mathrm{IP}^{2+}$ with $N_{\mathrm{C}} \gtrsim 30$ carbon atoms reported by Malloci et al. (2007b) is confirmed.

\subsection{Photoionization cross sections}

Experimental total action cross sections per carbon atom, $\sigma_{\mathrm{I}+\mathrm{F}}^{\mathrm{C}}$, were obtained following the procedure described in Appendix $\mathrm{C}$. The photoionization cross sections, $\sigma_{\mathrm{I}}^{\mathrm{C}}$, were then derived using the branching ratio depicted in Fig. 4. The $\sigma_{\mathrm{I}+\mathrm{F}}^{\mathrm{C}}$ curves are expected to provide a lower value for the absolute photoabsorption cross sections, $\sigma_{\text {abs }}^{\mathrm{C}}$ (see Eq. (C.1)). Since $\sigma_{\text {abs }}^{\mathrm{C}}$ of the studied cations could not be extracted from the performed experiment and have not been reported so far in the literature, we compare these curves with the theoretical photoabsorption cross sections, $\sigma_{\text {abs, theo }}^{\mathrm{C}}$, which were computed using time-dependent density functional theory (TD-DFT) in line with our previous work (Malloci et al. 2004, 2007a) and as described in Appendix E. All obtained cross sections, experimental and theoretical, are displayed in Fig. 5 and compared to each other in the following at high $(>14 \mathrm{eV})$ and low $(<14 \mathrm{eV})$ energies.

Above $14 \mathrm{eV}$, the cross sections are globally consistent (see Fig. 5). Still, the values of $\sigma_{\mathrm{I}+\mathrm{F}}^{\mathrm{C}}$ are found to be systematically larger than those of $\sigma_{\text {abs, theo }}^{\mathrm{C}}$ around the peak at $17 \mathrm{eV}$. In addition, there is a trend of increasing $\sigma_{\mathrm{I}+\mathrm{F}}^{\mathrm{C}}$ at the peak with molecular size. The case of $\mathrm{C}_{48} \mathrm{H}_{20}^{+}$has to be considered with caution though due to a less accurate calibration procedure (see Appendix C). Contrary to the experimental cross sections, the values of $\sigma_{\text {abs, theo }}^{\mathrm{C}}$ stay close to each other, which is expected from the proportionality of the photoabsorption cross sections with $N_{\mathrm{C}}$. Nevertheless, it is not yet possible to assess the precision of the calculated cross sections. Comparison with an experimental photoabsorption cross section at high energies (up to $30 \mathrm{eV}$ ) has so far only been done for neutral anthracene, $\mathrm{C}_{14} \mathrm{H}_{10}$ (Malloci et al. 2004). It is interesting to mention that this comparison reveals overall good agreement between the calculated and measured cross sections but with differences on the band positions and widths (in the theoretical spectra the band width is artificial). Furthermore, around the high-energy peak

http://astrochemistry.oa-cagliari.inaf.it/database/ observed at $18 \mathrm{eV}$, the discrepancy appears similar to the one illustrated in Fig. 5 in the cases of $\mathrm{C}_{32} \mathrm{H}_{14}^{+}$and $\mathrm{C}_{36} \mathrm{H}_{18}^{+}$.

Below $14 \mathrm{eV}, \sigma_{\mathrm{I}+\mathrm{F}}^{\mathrm{C}}$ and $\sigma_{\mathrm{I}}^{\mathrm{C}}$ are comparable since the fragmentation is negligible. A plateau is observed from 11.3 to $12.9 \mathrm{eV}$ in these experimental cross sections whereas the theoretical photoabsorption cross section exhibits strong bands (see Figs. 5 and 6). Ionization cross sections in PAHs may also display considerable structure, corresponding both to autoionization resonances and to the opening of channels as energy increases, making additional excited states accessible in the resulting ion with one electron less, i.e., vertical transitions (see e.g., Bréchignac et al. 2014).

Theoretical considerations show that the orbital picture of ionization involving valence one-electron bands is severely contaminated by shake-up states, which involve two electrons, one promoted to an excited bound state and the other to the ionization continuum (Deleuze et al. 2001). The authors have shown that in the case of $\pi$ orbitals, this happens at energies as low as $8 \mathrm{eV}$ for the first ionization (case of neutral PAHs). The density of excited states quickly becomes very large, blending in a quasi-continuum as energy increases (Deleuze et al. 2001). In addition, each electronic excited state may display vibrational structure on top of it, i.e., vibronic states, producing further structure on a smaller scale. Qualitatively, a jump in the ionization cross section is therefore expected when a major channel becomes accessible via a valence one-electron transition, followed by a long plateau-like tail produced by all states coupling to it due to electronic correlation and vibronic coupling. On top of this, there may be electronic transitions to excited states of the parent molecule which have low coupling with the ionization continuum, therefore preferentially relaxing via radiationless transitions to lower electronic states. These do not show in the ionization cross sections. The theoretical method used here to compute the absolute, total cross section does not distinguish among different categories of electronic excitation; they are in principle all included together (except vibronic coupling) and cannot be distinguished in these calculations. More complex techniques involving many-body theory can compute the structure of the ionization cross sections with considerable accuracy (see e.g., Baiardi et al. 2017, for a recent review). However, their computational cost would be extremely high for the species considered here, and they are out of the scope of the present work, in which such detailed structure is not resolved in any case.

Below $14 \mathrm{eV}$, it is clear that there is a part of the photoabsorption cross section that does not lead to ionization, which we referred to as $\sigma^{\prime}$ in Eq. (C.1). These correspond to excitations which involve fast relaxation via a strong coupling between electronic states and with nuclear states. This leads to vibrational excitation of the parent ion, which is expected to subsequently relax its energy by radiative cooling since no fragmentation is observed. At energies higher than $14 \mathrm{eV}$, evidence for such transitions, if they exist, is hindered by the precision of our experimental and theoretical data, as discussed above.

\section{Astrophysical recipes}

\subsection{Charge state of astro-PAHs}

Several modeling studies on the charge state of astro-PAHs have considered that these species could reach the dication and marginally the trication states (Bakes et al. 2001; Weingartner \& Draine 2001a; Andrews et al. 2016). In Fig. 7, we compiled the ionization potentials $\left(\mathrm{IP}^{(Z+1)+}\right)$ from neutral to cation $(Z=0)$, 

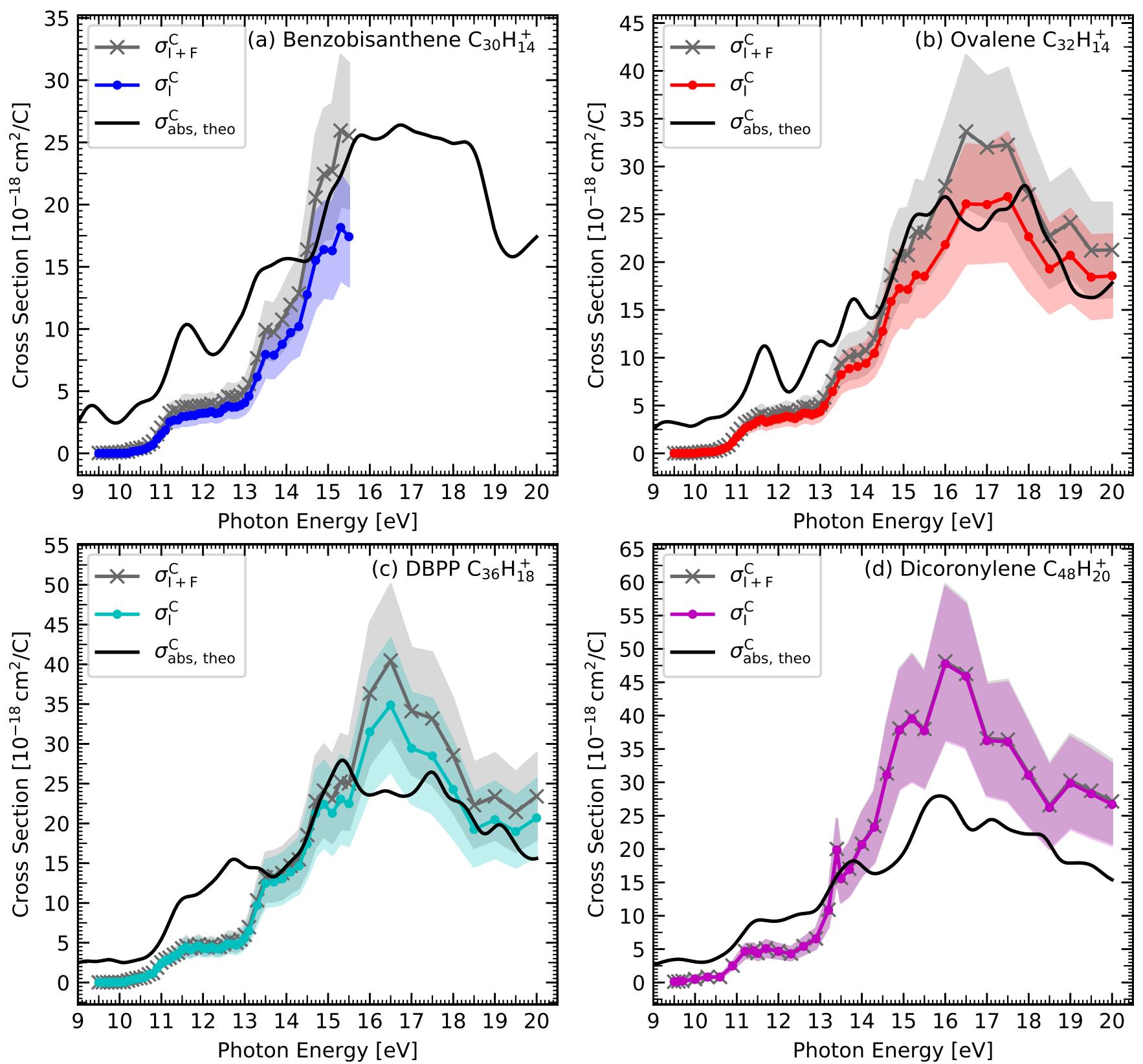

Fig. 5. Experimentally obtained total action and ionization cross sections per $\mathrm{C}$ atom, $\sigma_{\mathrm{I}+\mathrm{F}}^{\mathrm{C}}$ and $\sigma_{\mathrm{I}}^{\mathrm{C}}$, respectively, of the studied PAH cations as a function of photon energy, for (a) benzobisanthene, (b) ovalene, (c) DBPP, and (d) dicoronylene after absorption of a single VUV photon, compared to their theoretical photoabsorption cross sections, computed by TD-DFT, per $\mathrm{C}$ atom, $\sigma_{\text {abs, theo }}^{\mathrm{C}}$.

cation to dication $(Z=1)$, and dication to trication $(Z=2)$, which were obtained from calculations (Malloci et al. 2007b), as well as $\mathrm{IP}^{\exp }$ and $\mathrm{AE}^{(\mathrm{Z}+1)+}$ from experiments (Clar et al. 1981; Hager \& Wallace 1988; Zhen et al. 2016, and this work). We compared this data set with two analytical descriptions. Both start from a classical model of the energy it takes to remove one electronic charge from a small, conducting particle. Weingartner \& Draine (2001b) considered conducting spheres, and added an empirical correction term to account for both quantum effects and PAH geometry, which is planar and not spherical. This additional term was determined to fit a set of data on first and second ionization potentials of PAHs and led to

$\mathrm{IP}_{\mathrm{WD}}^{(Z+1)+}=W+\frac{1}{4 \pi \varepsilon_{0}}\left[\left(Z+\frac{1}{2}\right) \frac{e^{2}}{a}+(Z+2) \frac{e^{2}}{a} \frac{0.03 \mathrm{~nm}}{a}\right] \frac{1 \mathrm{C}}{e}$, where $Z$ is the ion charge, $\varepsilon_{0}$ is the vacuum permittivity in $\mathrm{F} \cdot \mathrm{nm}^{-1}, e$ is the elementary charge in $\mathrm{C}, W$ is the work function of bulk graphite, $W=4.4 \mathrm{eV}$, and $a$, the effective radius in $\mathrm{nm}$, is proportional to $N_{\mathrm{C}}$ via the relation

$a=\sqrt[3]{\frac{N_{\mathrm{C}}}{468}}$

In Eq. (1), the first term in square brackets corresponds to the classical conducting sphere model and the second term is the empirical correction. For the data set reported in Fig. 7, we find that, instead of $W=4.4 \mathrm{eV}$, a value of $W=3.9 \mathrm{eV}$ leads to a better fit (black curves in Fig. 7). A satisfactory model is obtained for all $Z$ values considered $(Z=0,1,2)$. The empirical formula which was adjusted for $Z=0,1$ appears also to be appropriate for 


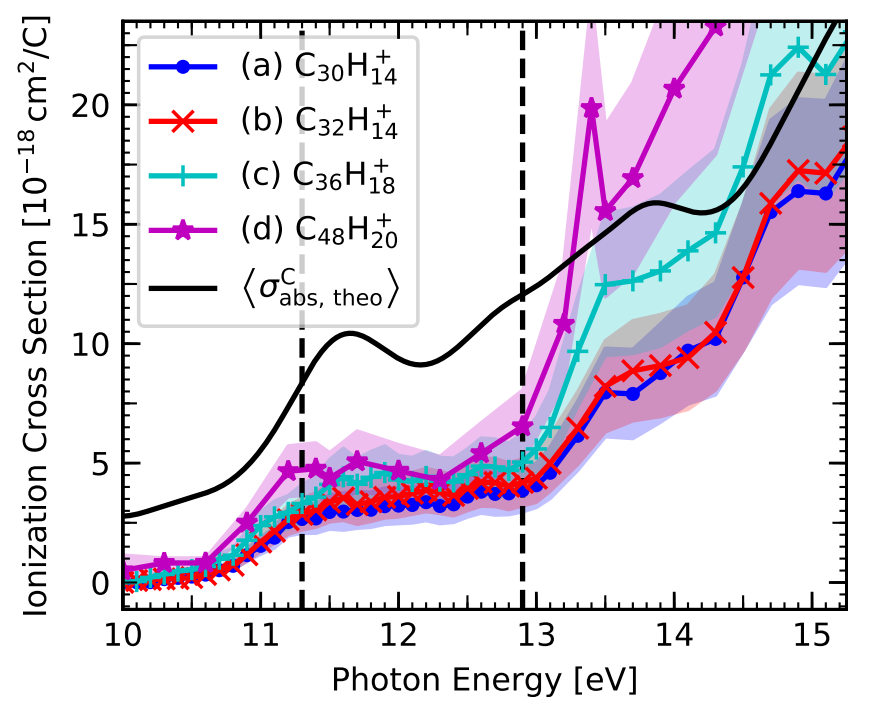

Fig. 6. Experimentally obtained photoionization cross sections per $\mathrm{C}$ atom, $\sigma_{\mathrm{I}}^{\mathrm{C}}$, of the studied PAH cations as a function of photon energy, (a) benzobisanthene, (b) ovalene, (c) DBPP, and (d) dicoronylene after absorption of a single VUV photon. The average of the calculated photoabsorption cross sections, $\left\langle\sigma_{\mathrm{abs}, \text { theo }}^{\mathrm{C}}\right\rangle$, is also presented. The dashed vertical lines mark the transitions between the different ionization regimes.

$Z=2$. The adjustment that we made on the $W$ value corresponds to a vertical shift and somehow depends on the considered data set. For instance, we can see that our reported values for $\mathrm{AE}^{(Z+1)+}$ are systematically slightly above the DFT values (cf. Table 1). Still, our derived value for $W$ appears in line with the values of about $4.0 \mathrm{eV}$, which were calculated for similar-sized PAHs by Kvashnin et al. (2013).

The second formalism to describe $\mathrm{IP}^{(Z+1)+}$ is given by Bakes $\&$ Tielens (1994b) who considered a thin conducting disk instead of a sphere, yielding

$\mathrm{IP}_{\mathrm{BT}}^{(Z+1)+}=W+\left(Z+\frac{1}{2}\right) \frac{25.1}{\sqrt{N_{\mathrm{C}}}}$.

The gray curves in Fig. 7 were obtained from Eq. (3) (Bakes $\&$ Tielens 1994b) and using $W=3.9 \mathrm{eV}$, as derived above. The curve provides a very satisfactory description of the data for $Z=0$, but tends to increasingly fail for higher $Z$ values. This trend was noticed by Weingartner \& Draine (2001b) and we can see that the discrepancy even increases for $Z=2$. Tuning the value of $\mathrm{W}$ does not change the shape of the curves and this emphasizes the need to include quantum effects in the estimation of $\mathrm{IP}^{(Z+1)+}$

We can see from Fig. 7 and Eq. (1) that a fraction of the photons absorbed in $\mathrm{HI}$ regions can induce ionization of PAH cations. Taking the absorption and ionization cross sections shown in Fig. 5 and considering the radiation field of the prototypical NGC 7023 NW PDR (Joblin et al. 2018), we can estimate that typically one photon over three absorbed in the [10-13.6] eV range by PAH cations with $N_{\mathrm{C}}=30-36$ will lead to ionization. The fraction of ionizing events will increase with increasing molecular size as the ionization potential shifts to lower energies. It reaches 0.5 for $\mathrm{C}_{48} \mathrm{H}_{20}^{+}$. We also note that the formation of $\mathrm{C}_{60}^{2+}$ will be more difficult to achieve than that of a $\mathrm{PAH}^{2+}$ of similar size, since the corresponding cations have relatively similar absorption cross sections but the value of $\mathrm{AE}^{2+}$ for

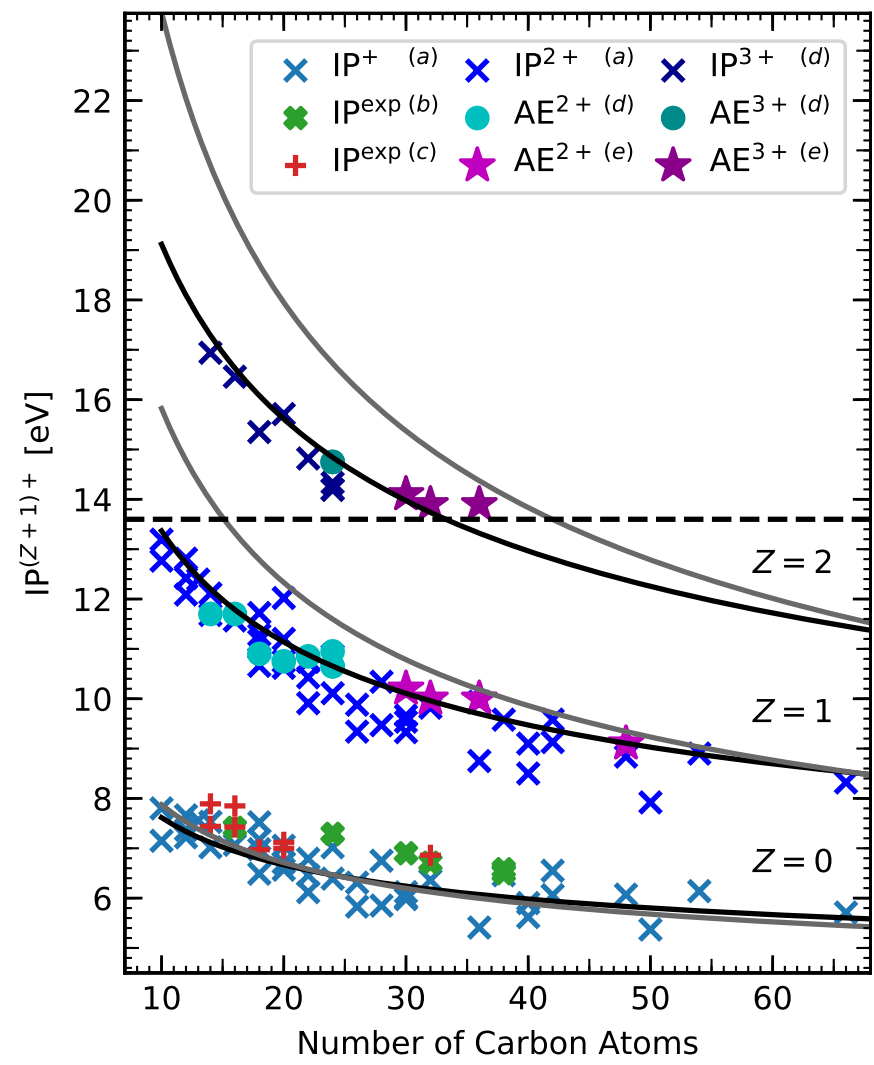

Fig. 7. Theoretically calculated ionization potentials, $\mathrm{IP}^{(Z+1)+}$, experimentally obtained $\mathrm{IP}^{\mathrm{exp}}$ and appearance energies, $\mathrm{AE}^{(Z+1)+}$, as a function of the number of carbon atoms. For $Z=0,1,2$, the values refer to the transition $\mathrm{PAH}^{(Z)+} \rightarrow \mathrm{PAH}^{(Z+1)+}$. The black and gray curves are the modeled $\mathrm{IP}_{\mathrm{WD}}^{(Z+1)+}$ from Weingartner \& Draine (2001b) and $\mathrm{IP}_{\mathrm{BT}}^{(Z+1)+}$ from Bakes \& Tielens (1994b), respectively, as an estimate of the $\operatorname{IP}^{(Z+1)+}$ evolution as a function of PAH size and charge, $Z$ (see Eqs. (1) and (3) and text for details). The dashed horizontal line marks the $13.6 \mathrm{eV}$ photon energy cut-off for $\mathrm{H}$ I regions. Notes: (a) taken from Malloci et al. (2007b); (b) taken from Clar et al. (1981); (c) taken from Hager \& Wallace (1988); (d) taken from Zhen et al. (2016); (e) this work.

$\mathrm{C}_{60}^{+}$is significantly higher, $(10.5 \pm 0.1) \mathrm{eV}$ (Douix et al. 2017), compared to $8.7 \mathrm{eV}$ for a $\mathrm{PAH}^{+}$with $N_{\mathrm{C}}=60$ (see Fig. 7).

\subsection{Photoionization yield}

Photoionization yields of PAH cations were derived for all studied species by dividing the experimental photoionization cross section, $\sigma_{\mathrm{I}}^{\mathrm{C}}$, by the theoretical photoabsorption cross section, $\sigma_{\text {abs, theo }}^{\mathrm{C}}$. In Sect. 3.2, we discussed the precision of both the experimental and theoretical cross sections. This can impact the photoionization yields. At energies below $14 \mathrm{eV}$, the presence of bands in $\sigma_{\text {abs, theo }}^{\mathrm{C}}$, which are not present in $\sigma_{\mathrm{I}}^{\mathrm{C}}$, can induce spectral features in the photoionization yields (e.g., the $12 \mathrm{eV}$ peak obtained for $\mathrm{C}_{32} \mathrm{H}_{14}^{+}$), which are as precise as the calculated spectrum. Nevertheless, Fig. 8 shows that the photoionization yields display comparable features for the studied molecules, with a rise starting at the ionization thresholds, $\mathrm{AE}^{2+}$, the plateau in the $11.3-12.9 \mathrm{eV}$ range followed by another rise to reach the maximum value. There is some uncertainty on this maximum value because of the unknown contribution from $\sigma^{\prime}$ (cf. Sect. 3.2). In the following, we made the hypothesis that the contribution of $\sigma^{\prime}$ at high energies $(20 \mathrm{eV})$ is minor and that the photoionization yields are limited by the photoionization BR, which never 


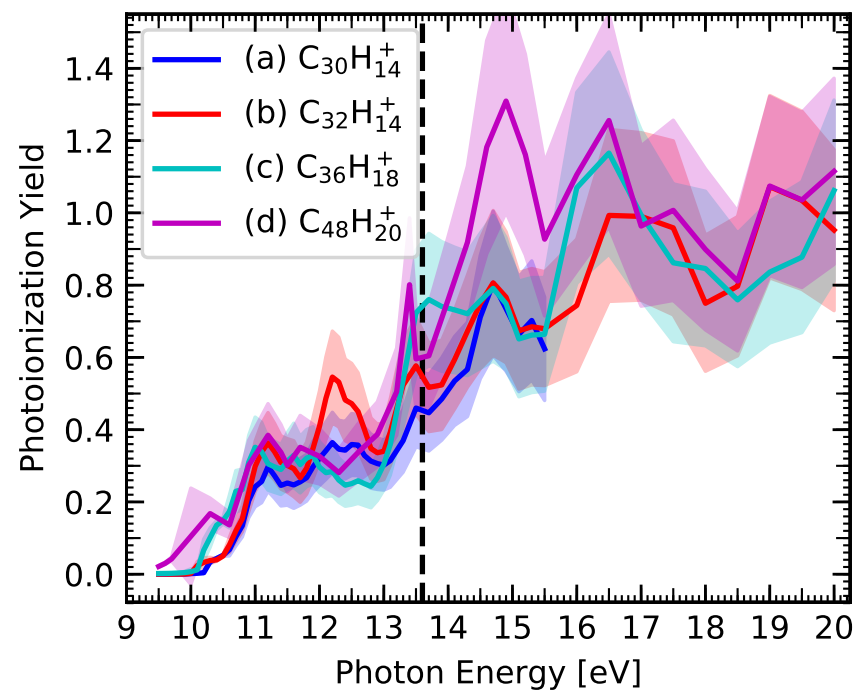

Fig. 8. Photoionization yields of the studied cations derived from the ionization and absorption cross sections (see Fig. 5) and scaled as explained in Sect. 4.2. The dashed vertical line marks the $13.6 \mathrm{eV}$ photon energy cut-off for $\mathrm{H}$ I regions.

reaches unity as shown in Fig. 4. The mean values at high energies of the photoionization yields were therefore scaled using the ionization $\mathrm{BR}$ at $20 \mathrm{eV}$. The resulting curves are presented in Fig. 8.

Data on the photoionization yields of neutral PAHs were derived in experimental studies performed by Verstraete et al. (1990) and Jochims et al. (1996). The latter authors proposed a rule of thumb to facilitate the implementation of this yield into models. This consists of a linear function of the photon energy with dependence on the ionization potential. On the basis of our results, we propose the use of a similar approach to describe the evolution of the photoionization yield of PAH cations with molecular size. The resulting function, $Y_{\text {ion }}^{+}$, is based on the above described ionization regimes which occur in different energy ranges (values in $\mathrm{eV}$ ) as

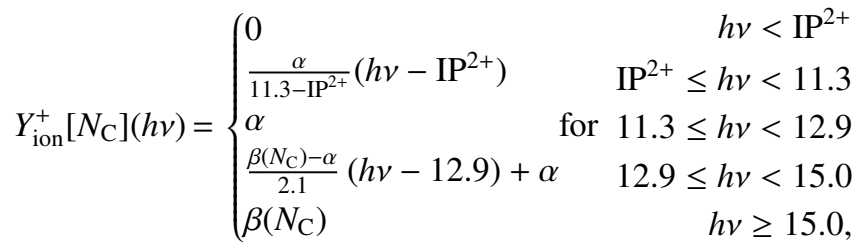

where $\alpha=0.3$ is the value of the plateau and $\beta$ depends on $N_{\mathrm{C}}$ with

$\beta\left(N_{\mathrm{C}}\right)=\left\{\begin{array}{lrr}0.59+8.1 \times 10^{-3} N_{\mathrm{C}} & \text { for } & 32 \leq N_{\mathrm{C}}<50 \\ 1 & & N_{\mathrm{C}} \geq 50 .\end{array}\right.$

The reported $\beta$ values represent the values at $20 \mathrm{eV}$ of the ionization BR (Fig. 4). These can be considered as maximum values since they neglect a possible contribution of $\sigma^{\prime}$ to the photoabsorption cross section as discussed above. These values were found to increase linearly with size for the studied size range with the dependence given by Eq. (5). Extrapolation to larger sizes leads to a $\beta$ value of 1 for $N_{\mathrm{C}} \geq 50$. This trend differs from the case of neutral PAHs for which Jochims et al. (1996) concluded that $\beta=1$ is independent of size, in agreement with previous measurements by Verstraete et al. (1990).

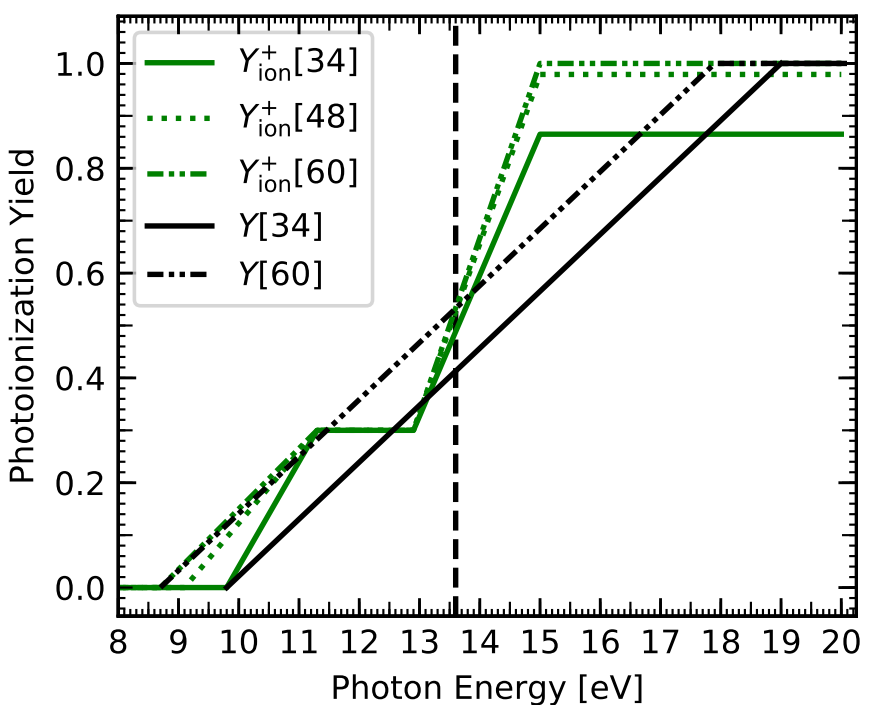

Fig. 9. Photoionization yields, $Y_{\mathrm{ion}}^{+}\left[N_{\mathrm{C}}\right](h v)$, calculated from Eqs. (4) and (5) for PAH cations with $N_{\mathrm{C}}=34,48$, and 60 atoms. The photoionization yields, $Y\left[N_{\mathrm{C}}\right](h v)$, for $N_{\mathrm{C}}=34$ and 60 , are displayed for comparison. These latter were estimated using Eqs. (4) and (5) from Jochims et al. (1996) adapted for neutral PAHs but taking into account the shift of the ionization potential to $\mathrm{IP}^{2+}$, which is relevant for cations. The dashed vertical line marks the $13.6 \mathrm{eV}$ photon energy cut-off for $\mathrm{HI}$ regions.

Figure 9 displays examples of $Y_{\text {ion }}^{+}\left[N_{\mathrm{C}}\right](h v)$ which were calculated from Eqs. (4) and (5), illustrating the variability of $Y_{\text {ion }}^{+}\left[N_{\mathrm{C}}\right](h v)$ with molecular size. No significant variation of this yield is expected for PAH cations with $N_{\mathrm{C}} \geq 50$. In their PAH evolution model, Andrews et al. (2016) considered the yield of PAH cations based on the recipe given by Jochims et al. (1996) for neutrals but taking into account the appropriate photoionization potential for cations, that is, values of $\mathrm{IP}^{2+}$. To illustrate the impact that this approximation may have on the model results, we report in Fig. 9 these estimated yields and compare them with our recommended yields by integrating from $\mathrm{IP}^{2+}$ to $13.6 \mathrm{eV}$. We find that for the medium-sized PAHs, as represented by $N_{\mathrm{C}}=34$, our integrated yield is larger by $19 \%$ compared to the previously available one, whereas for large PAHs, as represented by $N_{\mathrm{C}}=60$, it is smaller by $14 \%$. However, these simple estimates are inconclusive and models have to be run to evaluate the impact on the ionization of the PAH population in specific environments.

\section{Conclusion}

We studied the interaction of trapped PAH cations with VUV photons in the range from 9 to $20 \mathrm{eV}$, also covering photon energies present in HII regions and ionization fronts. Our experimental results provide a wealth of information on both ionization and fragmentation processes. The present article is focused on the detailed analysis of ionization, whereas fragmentation will be the subject of a future, dedicated work. Our initial goal was to explore the properties of large species for $N_{C}$ up to about 80 atoms. However, we were only able to obtain measurements on molecular sizes from 30 to 48 carbon atoms due to the very low solubility of large PAHs. Nevertheless, studies in this range allow us to access the major trends in the ionization properties of PAH cations due to a molecular size increase. Our findings can be summarized as follows. 
(i) Below $13.6 \mathrm{eV}$, the formation of a hot ion with subsequent (radiative) cooling is the major relaxation channel, followed by ionization whose yield reaches about 0.5 at $13.6 \mathrm{eV}$. From a molecular physics point of view, the yield comprises an interesting plateau at a value of 0.3 that extends over the energy range from 11.3 to $12.9 \mathrm{eV}$. This plateau reveals a spectral range in which there is strong competition between electronic and nuclear states. It would be interesting to investigate the dynamics of the relaxation of excited electronic states in this range using femtosecond (fs) pump-probe experiments (Marciniak et al. 2015).

(ii) Contrary to previous studies on neutrals, we were not able to observe that the photoionization yield reaches a value of 1 at high energies. At $20 \mathrm{eV}$, some dissociation is observed for all studied PAH cations, implying that the maximum of the yield cannot be larger than the branching ratio between ionization and dissociation, which increases with molecular size and reaches 0.98 for the largest studied ion, $\mathrm{C}_{48} \mathrm{H}_{20}^{+}$. In addition, we have not included a possible contribution in the photoabsorption events of the formation of a hot ion that would subsequently relax by radiative cooling in isolated conditions. This contribution would further lower the values of the photoionization yield. We have no explanation for the difference observed between neutrals and cations. Whether this is due to a change in their respective properties or the fact that experiments like ours using ion trapping are more sensitive to quantify this effect than experiments carried out on neutrals with different techniques is beyond the scope of this study but would be interesting to investigate.

Concerning astrophysical applications, we provide recipes to determine both the ionization potential and the photoionization yield of PAH cations as a function of their molecular size, which can be extended to larger sizes (typically $N_{\mathrm{C}}=100$ ). This yield can be combined with photoabsorption cross sections that are readily available from calculations using TD-DFT. All this molecular data can be used in models that describe the chemical evolution of PAHs in astrophysical environments. The range of photon energy we studied makes it possible to tackle the evolution of PAHs in extreme astronomical environments such as $\mathrm{HII}$ regions and ionization fronts. Observations of PAHs in these environments have so far been scarce due to their technical difficulty, but will become much more accessible thanks to the unique capabilities of the forthcoming James Webb Space Telescope (JWST).

As another example, the cavity around the star in NGC 7023 is expected to be an environment in which large $\mathrm{PAH}^{+}$and $\mathrm{PAH}^{2+}$ are present (Andrews et al. 2016; Croiset et al. 2016). The presence of dications is expected to impact both the heating of the gas by photoelectric effect and the AIB emission. Some initial IR action spectra of large PAH cations and dications were recorded by Zhen et al. (2017, 2018). These latter authors provide encouraging results suggesting that large ionized PAHs are good candidates for carrying the AIBs. Whether or not the spectral differences between cations and dications will be sufficient to differentiate both charge states in the observations nevertheless remains unclear. Still, we can predict that a detailed modeling approach combined with the wealth of spectral and spatial information, which will be delivered soon by the JWST, will be able to highlight the charge evolution of the PAH population and its impact on the physics and chemistry of PDRs.

Acknowledgements. This paper is dedicated to Sydney Leach, a great scientist who has been a pioneer in laboratory astrophysics and a major source of inspiration for the whole field. We are grateful to the staff from SOLEIL for the smooth running of the facility. We also wish to acknowledge the insightful comments of the referee on an earlier version of this paper. We acknowledge funding from the European Research Council under the European Union's Seventh Framework Programme ERC-2013-SyG, Grant Agreement no. 610256 NANOCOSMOS. This work was also supported by the Agence Nationale de la Recherche (France), under project number ANR-08-BLAN-0065. G. W. thanks the European Union (EU) for support under the Horizon 2020 framework for the Marie Skłodowska-Curie action EUROPAH, Grant Agreement no. 722346. S.Q. and D.P. acknowledge financial support from the Spanish Agencia Estatal de Investigación (MAT2016-78293-C6-3-R; AEI/FEDER, UE), Xunta de Galicia (Centro Singular de Investigación de Galicia accreditation 2016-2019, ED431G/09), the European Regional Development Fund-ERDF, and the European FET-OPEN project SPRING, Grant Agreement no. 863098. Finally, this project was granted access to the HPC resources at the CALMIP supercomputing centre under project P20027.

\section{References}

Andrews, H., Boersma, C., Werner, M. W., et al. 2015, ApJ, 807, 99

Andrews, H., Candian, A., \& Tielens, A. G. G. M. 2016, A\&A, 595, A23

Baiardi, A., Paoloni, L., Barone, V., Zakrzevski, V. G., \& Ortiz, J. V. 2017, J. Chem. Theory Comput., 13, 3120

Bakes, E. L. O., \& Tielens, A. G. G. M. 1994a, ASPC, 58, 412

Bakes, E. L. O., \& Tielens, A. G. G. M. 1994b, ApJ, 427, 822

Bakes, E. L. O., Tielens, A. G. G. M., \& Charles W. Bauschlicher, J. 2001, ApJ, 556, 501

Bréchignac, P., Garcia, G. A., Falvo, C., et al. 2014, J. Chem. Phys., 141, 164325

Cataldo, F., Ursini, O., Angelini, G., \& Iglesias-Groth, S. 2011, Fuller. Nanotub. Car. N., 19, 713

Clar, E., Robertson, J. M., Schloegl, R., \& Schmidt, W. 1981, J. Am. Chem. Soc., 103,1320

Compiègne, M., Abergel, A., Verstraete, L., et al. 2007, A\&A, 471, 205

Croiset, B. A., Candian, A., Berné, O., \& Tielens, A. G. G. M. 2016, A\&A, 590, A26

Deleuze, M. S., Trofimov, A. B., \& Cederbaum, L. S. 2001, J. Chem. Phys., 115, 5859

Douix, S., Duflot, D., Cubaynes, D., Bizau, J.-M., \& Giuliani, A. 2017, J. Phys. Chem. Lett., 8, 7

Giuliani, A., Giorgetta, J.-L., Ricaud, J.-P., et al. 2012, Nucl. Instrum. Methods Phys. Res. B, 279, 114

Hager, J. W., \& Wallace, S. C. 1988, Anal. Chem., 60, 5

Joblin, C., Bron, E., Pinto, C., et al. 2018, A\&A, 615, A129

Jochims, H. W., Baumgaertel, H., \& Leach, S. 1996, A\&A, 314 1003

Kvashnin, D. G., Sorokin, P. B., Brüning, J. W., \& Chernozatonskii, L. A. 2013, Appl. Phys. Lett., 102, 183112

Le Page, V., Snow, T. P., \& Bierbaum, V. M. 2003, ApJ, 584, 316

Malloci, G., Mulas, G., \& Joblin, C. 2004, A\&A, 426, 105

Malloci, G., Joblin, C., \& Mulas, G. 2007a, Chem. Phys., 332, 353

Malloci, G., Joblin, C., \& Mulas, G. 2007b, A\&A, 462, 627

Marciniak, A., Despré, V., Barillot, T., et al. 2015, Nat. Commun., 6, 1

Matsuzawa, N. N., Ishitani, A., Dixon, D. A., \& Uda, T. 2001, J. Phys. Chem. A, 105,4953

Milosavljević, A. R., Nicolas, C., Gil, J.-F., et al. 2012, J. Synchrotron Rad., 19, 174

Montillaud, J., Joblin, C., \& Toublanc, D. 2013, A\&A, 552, A15

Nahon, L., de Oliveira, N., Garcia, G. A., et al. 2012, J. Synchrotron Rad., 19, 508

Tancogne-Dejean, N., Oliveira, M. J. T., Andrade, X., et al. 2020, J. Chem. Phys., 152,124119

Tielens, A. G. G. M. 2005, The Physics and Chemistry of the Interstellar Medium (Cambridge: Cambridge University Press)

Verstraete, L., Leger, A., D'Hendecourt, L., Defourneau, D., \& Dutuit, O. 1990, A\&A, 237, 436

Vicente, S., Berné, O., Tielens, A. G. G. M., et al. 2013, ApJ, 765, L38

Visser, R., Geers, V. C., Dullemond, C. P., et al. 2007, A\&A, 466, 229

Weingartner, J. C., \& Draine, B. T. 2001a, ApJS, 134, 263

Weingartner, J. C., \& Draine, B. T. 2001b, ApJ, 548, 296

Yabana, K., \& Bertsch, G. F. 1996, Phys. Rev. B, 54, 4484

Zhen, J., Castellanos, P., Paardekooper, D. M., et al. 2015, ApJ, 804, L7

Zhen, J., Rodriguez Castillo, S., Joblin, C., et al. 2016, ApJ, 822, 113

Zhen, J., Castellanos, P., Bouwman, J., Linnartz, H., \& Tielens, A. G. G. M. 2017, ApJ, 836, 28

Zhen, J., Candian, A., Castellanos, P., et al. 2018, ApJ, 854, 27 
Appendix A: Synthesis of dicoronylene, $\mathrm{C}_{48} \mathrm{H}_{20}$

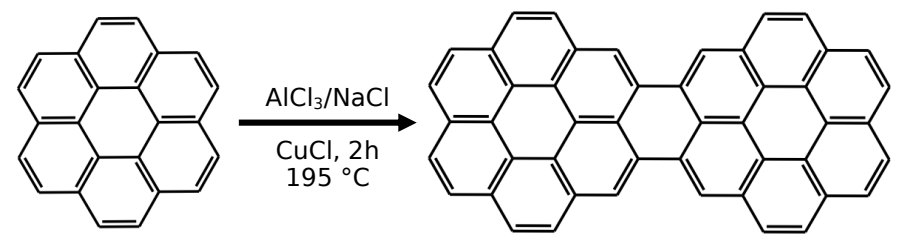

Fig. A.1. Schematic of the synthesis of dicoronylene, $\mathrm{C}_{48} \mathrm{H}_{20}$, from coronene, $\mathrm{C}_{24} \mathrm{H}_{12}$.

Dicoronylene was synthesized following the procedure reported by Cataldo et al. (2011). A mixture of coronene $(0.060 \mathrm{~g}$, $0.199 \mathrm{mmol}), \mathrm{AlCl}_{3}(4.20 \mathrm{~g}, 31.2 \mathrm{mmol}), \quad \mathrm{NaCl}(0.817 \mathrm{~g}$, $14.0 \mathrm{mmol})$, and $\mathrm{CuCl}(0.043 \mathrm{~g}, 0.440 \mathrm{mmol})$ was stirred at $195^{\circ} \mathrm{C}$ for $2 \mathrm{~h}$. Subsequently, an aqueous solution of $\mathrm{HCl}(10 \%$, $20 \mathrm{ml}$ ) was slowly added resulting in the precipitation of a red solid. This solid was filtrated and washed with aqueous solution of $\mathrm{HCl}(10 \%, 20 \mathrm{ml})$, hot water $(20 \mathrm{ml})$ and acetone $(3 \times 20 \mathrm{ml})$. The resulting solid was dried to obtain dicoronylene as a red solid (54 mg, 40\%).

\section{Appendix B: Action spectra scaling procedure}

From the recorded mass spectra, peak intensities of parent ions and their photoproducts can be deduced. The secondary ions produced upon VUV irradiation consist of the dication with peak intensity $S_{\mathrm{I}}$, and the photofragments with summed peak intensity $S_{\mathrm{F}}$, including the $\mathrm{H}$ and $2 \mathrm{H} / \mathrm{H}_{2}$ loss channels. Due to detector characteristics, doubly ionized molecules are detected more efficiently than singly ionized molecules. Therefore, the peak intensities derived from the mass spectra have to be scaled by the detector gain efficiency, $\varepsilon$, to retrieve values that scale with abundances. Thermo Scientific ${ }^{\mathrm{TM}}$ provides $\varepsilon_{+}=0.29$ for parent ions and fragments, $\varepsilon_{2+}=0.42$ for dications and a value of 0.54 for trications. There is some gain change with mass but this is a minor correction for the range of studied masses.

The total number of ions in the trap, $P_{0}$ (in uncalibrated values), can then be calculated with

$P_{0}=\frac{P_{t}}{\varepsilon_{+}}+\frac{S_{\mathrm{F}}}{\varepsilon_{+}}+\frac{S_{\mathrm{I}}}{\varepsilon_{2+}}$,

where $\frac{P_{t}}{\varepsilon_{+}}$is the number of parent ions after irradiation time, $t$. Building the action spectra requires to derive normalized photoproduct intensities for $S_{\mathrm{I}}$ and $S_{\mathrm{F}}$, which can be obtained by first dividing them by $P_{0}$ and then correcting for the variation of the photon flux, $\Phi(v)$. Indeed the latter evolved in energy due to spectral shape variations. Changes were also made in the irradiation time, $t$, and monochromator exit slit width, $s$, so that the total photoproduct intensity remains smaller than $c a$. $12 \%$ of $P_{t}$, as seen in the mass spectra. This led to

$S_{\mathrm{I}, \mathrm{F}}^{\mathrm{norm}}(v)=\frac{S_{\mathrm{I}, \mathrm{F}}(v)}{\Phi_{\mathrm{norm}}(v) t(v) s(v)}$,

where $\Phi_{\text {norm }}(v)$ is normalized to be 1 at its maximum at $9.5 \mathrm{eV}$. We note that the obtained intensities are in arbitrary units (see Fig. 3) and not in percentage of the total number of ions because of the scaling by the relative photon flux.

\section{Appendix C: Photoproduct cross sections scaling procedure}

The absorption of a photon by the parent ion leads to different relaxation channels. Considering monochromatic radiation, the photoabsorption cross section, $\sigma_{\mathrm{abs}}$, can therefore be decomposed into the sum of the cross sections for each relaxation channel as

$\sigma_{\mathrm{abs}}=\sigma_{\mathrm{I}+\mathrm{F}}+\sigma^{\prime}$

where $\sigma_{\mathrm{I}+\mathrm{F}}$ is the cross section leading to the production of the secondary products with intensities $S_{\mathrm{I}}$ and $S_{\mathrm{F}}$ (see Appendix B), and $\sigma^{\prime}$ is the cross section for the creation of a hot ion that will relax its internal energy by radiative cooling and/or collisions with buffer gas $(\mathrm{He})$ in our experiment. These processes cannot be traced in our experiment and only $\sigma_{\mathrm{I}+\mathrm{F}}$ can be estimated following

$\sigma_{\mathrm{I}+\mathrm{F}}=\frac{\gamma}{\Phi t} \ln \left(\frac{\varepsilon_{+} P_{0}}{P_{t}}\right)$

with the photon flux, $\Phi$, in photons $\mathrm{cm}^{-2} \mathrm{~s}^{-1}$, a form factor, $\gamma$, describing the overlap of the photon beam and the ion cloud, the total number of ions, $P_{0}$, and the number of parent ions, $\frac{P_{t}}{\varepsilon_{+}}$, after irradiation time, $t$. Plugging $P_{0}$ from Eq. (B.1) in Eq. (C.2), we get

$\sigma_{\mathrm{I}+\mathrm{F}}=\frac{\gamma}{\Phi t} \ln \left(1+\frac{S_{\mathrm{F}}}{P_{t}}+\frac{\varepsilon_{+} S_{\mathrm{I}}}{\varepsilon_{2+} P_{t}}\right)$

In order to determine cross sections in absolute units, the photon flux, $\Phi$, and the form factor, $\gamma$, have to be well known. Douix et al. (2017) managed to record the absolute photoionization cross section, $\sigma_{\mathrm{I}}^{\mathrm{C}_{60}^{+}}$, for the buckminsterfullerene cation, $\mathrm{C}_{60}^{+}$, by carefully measuring these parameters and applying Eq. (C.3), where the term $\frac{S_{\mathrm{F}}}{P_{t}}$ was zero due to the nondissociation of $\mathrm{C}_{60}^{+2}$. In our experiment, we trapped $\mathrm{C}_{60}^{+}$and recorded its dication peak under the same irradiation conditions used for our studied PAHs except dicoronylene as explained below. We were therefore able to derive the value of $\frac{\gamma}{\Phi t}$ and use this factor to obtain experimental values for $\sigma_{\mathrm{I}+\mathrm{F}}$ from the photoproduct evolutions of our PAHs according to Eq. (C.3). This scaling procedure yields reasonable cross section values only above the $\mathrm{AE}^{2+}$ of $\mathrm{C}_{60}^{+}$. We bypassed this limitation by scaling the action spectra (see Sect. 3.1 and Appendix B) to the cross sections and replacing the values below the $\mathrm{AE}^{2+}$ of $\mathrm{C}_{60}^{+}$with the values from the scaled action spectra. Finally, to simplify the comparison between species, we divide $\sigma_{\mathrm{I}+\mathrm{F}}$ of each $\mathrm{PAH}$ cation by its respective $N_{\mathrm{C}}$, yielding $\sigma_{\mathrm{I}+\mathrm{F}}^{\mathrm{C}}$.

We note that in this calibration procedure, dicoronylene required a specific treatment. Indeed this ion was studied in different conditions since both the syringe flow rate and the monochromator exit slit width, $s$, were increased in order to get a sufficient signal. In order to correct at best for these changes, we applied corrections to the $\frac{\gamma}{\Phi t}$ factor in Eq. (C.2) by assuming that not only the photon flux but also the beam overlap with the ion cloud scales linearly with $s$, the latter is likely disputable but this is the best we could do.

2 The absolute photoionization cross section of $\mathrm{C}_{60}^{+}, \sigma_{\mathrm{I}}^{\mathrm{C}_{60}^{+}}$, can be accessed at https://zenodo.org/record/1001072. 


\section{Appendix D: Error estimation}

Depending on the acquisition time, a few hundred, $N$, mass spectra, $x$, are recorded for each photon energy step. Averaging these mass spectra for each photon energy yields one mean mass spectrum, $\bar{x}$, per photon energy with an absolute standard error of $\Delta \bar{x}=\frac{\sigma}{\sqrt{N}}$, where $\sigma$ is the standard deviation. The error bars, $\Delta f$, in Figs. 3 and 4 result from error propagation for a function, $f \rightarrow f\left(x_{1}, x_{2}, \ldots, x_{N}\right)$, according to

$\Delta f=\sqrt{\sum_{i=1}^{N}\left(\frac{\partial f}{\partial x_{i}} \Delta x_{i}\right)^{2}}$.

When determining the cross sections depicted in Fig. 5 following the procedure presented in Appendix $\mathrm{C}$ as well as the obtained photoionization yields shown in Figs. 8 and 9, the error on the cross section of $\mathrm{C}_{60}^{+}$, which was found to be below $21 \%$ by Douix et al. (2017) is used to propagate the errors.

\section{Appendix E: Theoretical cross section calculations}

Theoretical photoabsorption cross sections were obtained using TD-DFT, with the real-time, real-space method of Yabana \& Bertsch (1996), as implemented in the OcTOPUs computer code (Tancogne-Dejean et al. 2020). Not all species in this work were considered in our previous work (Malloci et al. 2007a). In addition, since here we need to use theoretical spectra up to relatively high energies $(\sim 20 \mathrm{eV})$, we also took the chance to verify at these energies the convergence of the calculations with respect to the simulation box size and grid spacing, the real-space equivalent of more conventional basis-set convergence for Gaussian-based
DFT for example. It is known (Matsuzawa et al. 2001) and mentioned in the OCTOPUS documentation that as a rule of thumb, larger simulation boxes are needed tof obtain converged values for the energies of higher lying electronic excited states, and for the intensities of the transitions involving them. Moreover, when using the real-time, real-space method implemented in OCTOPUS, the photoabsorption spectrum includes both transitions to discrete (bound) electronic states and to the continuum of unbound states. The former tend to converge to well-defined energies in the limit of infinite box size; the latter, instead, are artificially quantized by the boundary conditions of a finite simulation box, whose individual energies keep on changing with changing box size (and shape).

In this context, we started a computational effort to test convergence as a function of box size. This led us to conclude that the spectra in the database of Malloci et al. (2007a) are adequately converged for excitation energies up to $\sim 13 \mathrm{eV}$, meaning they are suitable for astronomical modeling purposes involving photons up to the Lyman limit. We instead had to consider substantially larger simulation box sizes and denser grid spacing to achieve convergence up to $\sim 20 \mathrm{eV}$, as required for this work. For our purposes, we found that a "minimal" simulation box can be achieved by considering the union of $8 \AA$ radius spheres centered on each atom of the molecule, or by a single larger sphere with an equivalent volume. In addition, a grid spacing of $0.18 \AA$ was needed to achieve convergence. All the calculations were performed on the OLYMPE supercomputer at the mesocentre CALcul en MIdi Pyrénées (CALMIP). We retained from the set of simulations two different converged spectra for each species and averaged them together in order to smooth out the contribution of free electron states and retain discrete ones. Finally, we

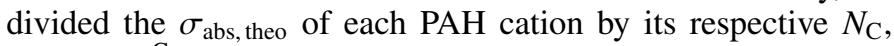
yielding $\sigma_{\text {abs, theo }}^{\mathrm{C}}$. 